\title{
Des monnaies en milieu rural : les découvertes du village de Courtisigny (les Fosses Saint-Ursin, Courseulles-sur-Mer, Calvados)
}

Coinage in rural areas: discoveries from the village of Courtisigny (les Fosses Saint-Ursin, Courseulles-sur-Mer, Calvados)

Münzen im ländlichen Raum: Die Funde aus dem mittelalterlichen Dorf von

Courtisigny „Les Fosses Saint-Ursin“ bei Courseulles-sur-Mer (Calvados, Normandie, Frankreich)

Monedas en medio rural: los descubrimientos de la aldea de Courtisigny ("Las fosas Saint-Ursin", Courseulles-sur-Mer, Calvados)

Pierre-Marie Guihard et Claire Hanusse

\section{(2)enEdition} Journals

Édition électronique

URL : https://journals.openedition.org/rao/3129

DOI : $10.4000 /$ rao.3129

ISBN : 978-2-7535-5014-8

ISSN : $1775-3732$

Éditeur

Presses universitaires de Rennes

Édition imprimée

Date de publication : 31 décembre 2015

Pagination : 401-413

ISBN : 978-2-7535-5012-4

ISSN : 0767-709X

\section{Référence électronique}

Pierre-Marie Guihard et Claire Hanusse, « Des monnaies en milieu rural : les découvertes du village de Courtisigny (les Fosses Saint-Ursin, Courseulles-sur-Mer, Calvados) », Revue archéologique de l'Ouest [En ligne], 32 | 2015, mis en ligne le 28 avril 2018, consulté le 18 août 2022. URL : http:// journals.openedition.org/rao/3129; DOI : https://doi.org/10.4000/rao.3129 


\title{
Des monnaies en milieu rural : les découvertes du village de Courtisigny (les Fosses Saint-Ursin, Courseulles-sur-Mer, Calvados)
}

\author{
Coinage in Rural Areas: Discoveries from the Village of Courtisigny \\ (les Fosses Saint-Ursin, Courseulles-sur-Mer, Calvados)
}

\author{
Pierre-Marie Guihard ${ }^{a}$ et Claire Hanusse ${ }^{b}$
}

\begin{abstract}
Résumé : Dans les années 1970 et 2000, les fouilles du village médiéval de Courtisigny (les « fosses Saint-Ursin », Courseulles-sur-Mer, Calvados) ont mis au jour un ensemble de monnaies antiques, médiévales et modernes. Ces découvertes ont fait l'objet d'une étude archéologique contribuant à une meilleure connaissance de leur circulation au cœur même du village. C'est à partir du croisement de données numismatiques et archéologiques qu'il est possible d'aborder la question de la monétarisation des campagnes entre le XII ${ }^{\mathrm{e}}$ et le XIV ${ }^{\mathrm{e}}$ siècles.

Abstract: The excavation of the Medieval village of Courtisigny (les "fosses Saint-Ursin", Courseulles-sur-Mer, Calvados) in 1970 and 2000 has brought to light a collection of Roman, Medieval and Modern coins. Their study has largely contributed to a better understanding of how they circulated even within the village itself. BY Comparing archaeological and numismatic data the question of the monetization of the rural areas between the $12^{\text {th }}$ and $14^{\text {th }}$ century will be broached.
\end{abstract}

Mots clés : monnaies, Moyen Âge, archéologie, circulation monétaire, monétarisation rurale.

Keywords: Coins, Middle Ages, archaeology, circulation of money, rural monetization.

Discrètes mais bien réelles, les découvertes monétaires effectuées lors des fouilles du village de Courtisigny (les Fosses Saint-Ursin, Courseulles-sur-Mer, Calvados) ne se caractérisent pas par leur abondance : quarante exemplaires ont été à ce jour enregistrés (tableau 1). Elles ne sont pas non plus à la source de trouvailles importantes sur le plan numismatique : il s'agit là d'un numéraire de faible valeur fiduciaire proche de la vie quotidienne, qui ne fait que rejoindre les observations réalisées sur le matériel issu d'autres fouilles de la même époque. Sans doute l'intérêt des découvertes monétaires de Courtisigny est-il ailleurs et particulièrement dans la présence de ces humbles vestiges épars sur le sol des habitations ou piégés dans les multiples éboulis identifiés en quelques points du site. Intérêt intrinsèque donc, lié à la contextualisation du numéraire et sa spatialisation. Intérêt plus général aussi, prompt à donner un autre relief à la vie économique des populations paysannes. En effet, peut-être est-il possible de voir en ce lot réduit le reflet d'une circulation plus ample en milieu rural. Aperçu fragile certes : du moins permet-il de constater l'importance relative de certaines émissions et leur évolution, bien loin du clivage académique opposant ville et campagne.

\footnotetext{
a Université de Caen Basse-Normandie, CRAHAM-UMR 6273, responsable du service de numismatique.

b Université de Caen Basse-Normandie, CRAHAM-UMR 6273.
} 


\begin{tabular}{|c|c|c|c|}
\hline \multirow{10}{*}{\begin{tabular}{|l|} 
Antiquité \\
\end{tabular}} & & Nombre & Total \\
\hline & Haut-Empire & & 3 \\
\hline & * Néron (54-68) & 1 & \\
\hline & ${ }^{*}$ Antonin-le-Pieux (138-161) & 1 & \\
\hline & *Indéterminée & 1 & \\
\hline & Bas-Empire & & 7 \\
\hline & * Constantin ler (307-337) & 3 & \\
\hline & * Magnence (350-353) & 1 & \\
\hline & *Époque théodosienne & 2 & \\
\hline & *Indéterminée & 1 & \\
\hline \multirow{22}{*}{$\begin{array}{l}\text { Moyen Âge et } \\
\text { temps modernes }\end{array}$} & Empire carolingien & & 1 \\
\hline & ${ }^{*}$ Louis le Pieux (814-840) & 1 & \\
\hline & Royaume de France & & 21 \\
\hline & * Louis VIII (1223-1226) ou Louis IX (1226-1270) & 1 & \\
\hline & * Philippe IV (1285-1314) & 5 & \\
\hline & * Charles IV (1322-1328) & 1 & \\
\hline & * Philippe VI (1328-1350) & 3 & \\
\hline & * Jean II (1350-1364) & 1 & \\
\hline & * Louis XI (1461-1483) & 2 & \\
\hline & * François ler (1515-1547) & 1 & \\
\hline & * Henri II (1547-1559) & 1 & \\
\hline & * Louis XIII (1610-1643) & 2 & \\
\hline & * Louis XIV (1643-1715) & 4 & \\
\hline & Monnayage baronnial & & 6 \\
\hline & ${ }^{*}$ Comté du Mans (1080-1130) & 1 & \\
\hline & * Comté de Vendôme (1180-1205) & 1 & \\
\hline & * Vicomté de Châteaudun (1200-1210) & 1 & \\
\hline & * Duché de Bretagne (1341-1364) / & & \\
\hline & imitations de monnaies françaises & 3 & \\
\hline & Royaume d'Angleterre & & 1 \\
\hline & ${ }^{*}$ Edouard II (1327-1377) & 1 & \\
\hline & Indéterminées Moyen Age & & 2 \\
\hline \multicolumn{2}{|r|}{ 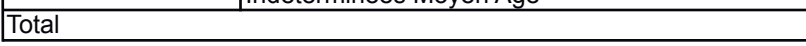 } & & 41 . \\
\hline
\end{tabular}

Tableau 1 : Composition de l'ensemble monétaire de Courtisigny (Courseulles-sur-Mer). Table 1: Composition of the coin assemblage of Courtisigny (Courseulles-sur-Mer).

\section{LE NUMÉRAIRE ANTIQUE...}

Attesté par dix exemplaires centrés sur le Haut-Empire (3) et le $\mathrm{IV}^{\mathrm{e}}$ siècle (7), le numéraire antique présente d'emblée un intérêt d'ordre chronologique (tableau 1). En effet, les monnaies romaines ont été mises au jour dans des ensembles archéologiques bien caractérisés du bas Moyen Âge et de l'époque moderne. Aucun exemplaire n'a été signalé dans les couches d'occupation les plus anciennes du gisement : l'ensemble est contemporain des périodes 2 et 3 (tableau 2). Il est encore malaisé d'interpréter avec le recul nécessaire la présence de ces monnaies. La documentation présentée au tableau 3 rassemble des découvertes issues de fouilles archéologiques. Au regard de ce recensement non exhaustif et limité au seul département du Calvados, il semble qu'un noyau de monnaies romaines soit fréquemment attesté sur des sites occupés au cours de la période médiévale. Pour certains auteurs, il y aurait là le signe d'une réutilisation de pièces beaucoup plus anciennes à des époques relativement proches de nous, comme cela a pu être notamment envisagé à propos des monnaies romaines déposées dans les ponts médiévaux de Cahors (Lot; Depeyrot, 1976) et comme le démontrent aussi nombre de découvertes signalées en Italie. En outre, le fait que certaines des monnaies romaines mises au jour à Courtisigny soient clairement associées à des niveaux d'occupation (cf. catalogue) confirmerait qu'elles étaient disponibles et utilisées.

Toutefois, l'hypothèse d'une réutilisation dans la circulation monétaire n'emporte pas la conviction. Nous savons qu'à Courtisigny et dans l'environnement immédiat du site, plusieurs occupations romaines ont été identifiées. C'est ainsi que l'on note la mise au jour en 1832 d'un dépôt renfermant plus de quatre mille monnaies du Bas-Empire au lieu-dit "Corfolands " situé à quelques centaines de mètres au nord de Courtisigny (Caumont, 1846, p. 437-439). Ailleurs, ce sont des sépultures gallo-romaines et des monnaies qui sont signalées à l'emplacement même du village médiéval (Doranlo, 1914, p. 411-412; en dernier lieu CAG 14, p. 66, notice 208). Dans ce contexte, les dix monnaies romaines pourraient tout simplement provenir de prélèvements de terre (pour l'enrichissement de jardins par exemple) réalisés sur des terrains occupés pendant l'Antiquité. Peut-être furent-elles alors pieusement conservées pour leur caractère exotique, voire folklorique plutôt qu'économique, par les inventeurs occasionnels ${ }^{1}$ ?

1. C'est aussi en ce sens que sont interprétées les découvertes de monnaies romaines réalisées sur l'habitat médiéval de Rougiers dans le Var : cf. Démians d'Archimbaud 1980, p. 266. 


\begin{tabular}{|c|c|}
\hline Période 2B fin XIle-début XIVe siècle & 0 \\
\hline Période 2C début XIVe-XIVe siècle & 24 \\
\hline Antiquité & 6 \\
\hline Empire carolingien & 1 \\
\hline Royaume de France / Bas Moyen Âge & 9 \\
\hline * Louis IX, Philippe IV, Philippe VI & \\
\hline Royaume de France / Post Moyen Âge & 2 \\
\hline * François ler, Henri II & \\
\hline Monnayage baronnial & 5 \\
\hline * Comté du Mans, Vicomté de Châteaudun, Duché de & \\
\hline Bretagne & \\
\hline Indéterminées Moyen Äge & 1 \\
\hline \multicolumn{2}{|l|}{ Période 2D fin XIVe-XVe siècle } \\
\hline Antiquité & 2 \\
\hline Royaume de France / Bas Moyen Äge & 4 \\
\hline * Philippe VI, Jean II, Louis XI & \\
\hline Royaume de France / Post Moyen Äge & 1 \\
\hline * Louis XIV & \\
\hline \multicolumn{2}{|l|}{ Période 3 Epoques moderne et contemporaine } \\
\hline Antiquité & 2 \\
\hline $\begin{array}{l}\text { Royaume de France / Post Moyen Äge } \\
\text { * Louis XIII, Louis XIV }\end{array}$ & 3 \\
\hline \multicolumn{2}{|l|}{ Espace ouvert XIIle-XXe siècle } \\
\hline Royaume de France / Bas Moyen Âge & 1 \\
\hline Royaume de France / Post Moyen Âge & 2 \\
\hline * Louis XIII, Gaston d'Orléans & \\
\hline Monnayage baronnial & 1 \\
\hline * Comté de Vendôme & \\
\hline Royaume d'Angleterre & 1 \\
\hline Total des monnaies & 41 \\
\hline
\end{tabular}

Tableau 2 : Périodisation de l'ensemble monétaire de Courtisigny (Courseulles-sur-Mer).

Table 2: Periodisation of the coin assemblage of Courtisigny (Courseulles-sur-Mer).

\section{2. ...MÉDIÉVAL ET MODERNE}

La série des pièces médiévales et modernes est plus significative. Avec trente et un exemplaires (tableau 1), elle représente certes un lot modeste, dont la continuité et la diversité, comme la répartition suivant les diverses époques d'occupation, n'en demeurent pas moins riches d'enseignements pour une meilleure connaissance du site et de la circulation monétaire en milieu rural.

Si les monnaies antérieures au $\mathrm{XII}^{\mathrm{e}}$ siècle demeurent anecdotiques sur le site de Courtisigny, il faut toutefois souligner la découverte - rare en contexte archéologique - d'un denier carolingien attribué à Louis le Pieux (814-840). Le contraste est flagrant avec la période immédiatement postérieure : remarquable par le nombre, le numéraire du bas Moyen Âge l'est aussi par son homogénéité dans le temps (tableau 1). Plus des trois quarts des monnaies se rattachent en effet à des émissions frappées entre le début du XII ${ }^{\mathrm{e}}$ siècle et le milieu du $\mathrm{XIV}^{\mathrm{e}}$ siècle. Il faut aussi souligner le lot formé par les monnaies de la Renaissance et de l'époque moderne. Bien caractérisées, celles-ci sont curieusement associées à des contextes spatialement très localisés (figure 1). Elles pourraient être mises en relation avec une fréquentation prolongée du site, dont l'abandon se situerait, d'après la documentation écrite et les découvertes archéologiques, entre le début du premier

\begin{tabular}{|l|c|c|c|}
\hline & Courtisigny & Rubercy & $\begin{array}{c}\text { Grentheville } \\
\text { (Trainecourt) }\end{array}$ \\
\hline Monnaies romaines & 9 & 4 & 17 \\
\hline $\begin{array}{l}\text { Monnaies médiévales et } \\
\text { modernes }\end{array}$ & 31 & 17 & 151 \\
\hline
\end{tabular}

Tableau 3 : Composition des monnaies isolées sur trois occupations médiévales du Calvados.

Table 3: Composition of the isolated coins found on the three Medieval sites in Calvados.

quart du XIV siècle et la fin du Xve siècle. Pour expliquer cet " abandon paisible " des lieux, l'hypothèse d'une récupération systématique des matériaux de construction a été avancée (Hanusse, 2006, p. 83). Parmi les dénominations représentées, les espèces se rattachent soit au système du denier tournois, soit à celui du denier parisis. Traditionnellement, le premier l'emporterait sur le second dans l'Ouest de la Normandie (voir les commentaires de Moesgaard, 2009, p. 256-259). Toutefois, cela n'apparait pas clairement dans la circulation monétaire de Courtisigny. Au cours du XIve siècle, les deux dénominations cohabitent dans des proportions presque identiques, sans que l'une ne prenne le pas sur l'autre.

Mais surtout, le lot monétaire de Courtisigny se caractérise par la prédominance quasi totale du monnayage royal et en particulier de Philippe IV (1285-1314) et de Philippe VI (1328-1350) dont l'activité monétaire se reflète dans la diversité des types mis au jour. Diversité inégale cependant comme le prouve la proportion des petites dénominations. Il apparaît en particulier que les petites espèces en billon noir ou " monnaies noires ${ }^{2}$ ", qui étaient destinées aux menues transactions de la vie quotidienne (Bompaire, 1999), sont ici très nombreuses. Si l'on confronte la part des monnaies blanches à celle des monnaies noires ${ }^{3}$, le résultat est patent : la domination des secondes sur les premières est tout à fait écrasante et démontre qu'elles étaient en circulation active sur le site. Ce phénomène est confirmé par d'autres découvertes isolées(tableau 4). Parmi les trouvailles enregistrées en Haute et Basse-Normandie, il est intéressant en effet de souligner que, à partir du second tiers du XIv ${ }^{e}$ siècle, les monnaies noires atteignent toujours des taux importants par rapport aux monnaies en or et aux monnaies blanches. On remarquera toutefois que les monnaies de forte valeur sortaient certainement moins des bourses et si elles étaient perdues, il est probable que l'usager s'efforçait de les retrouver.

2. Alliage d'argent à fort pourcentage de cuivre, ce qui donnait à la monnaie un aspect " noir».

3. La monnaie noire s'oppose à la monnaie blanche (gros et blancs), qui était d'une teneur en argent plus élevée, valant 20,12, 10, 6 et 5 deniers. Parmi les monnaies noires, les trésins, liards, hardis, doubles, deniers et oboles valaient respectivement $3,3,3,2,1$ et $1 / 2$ deniers. 


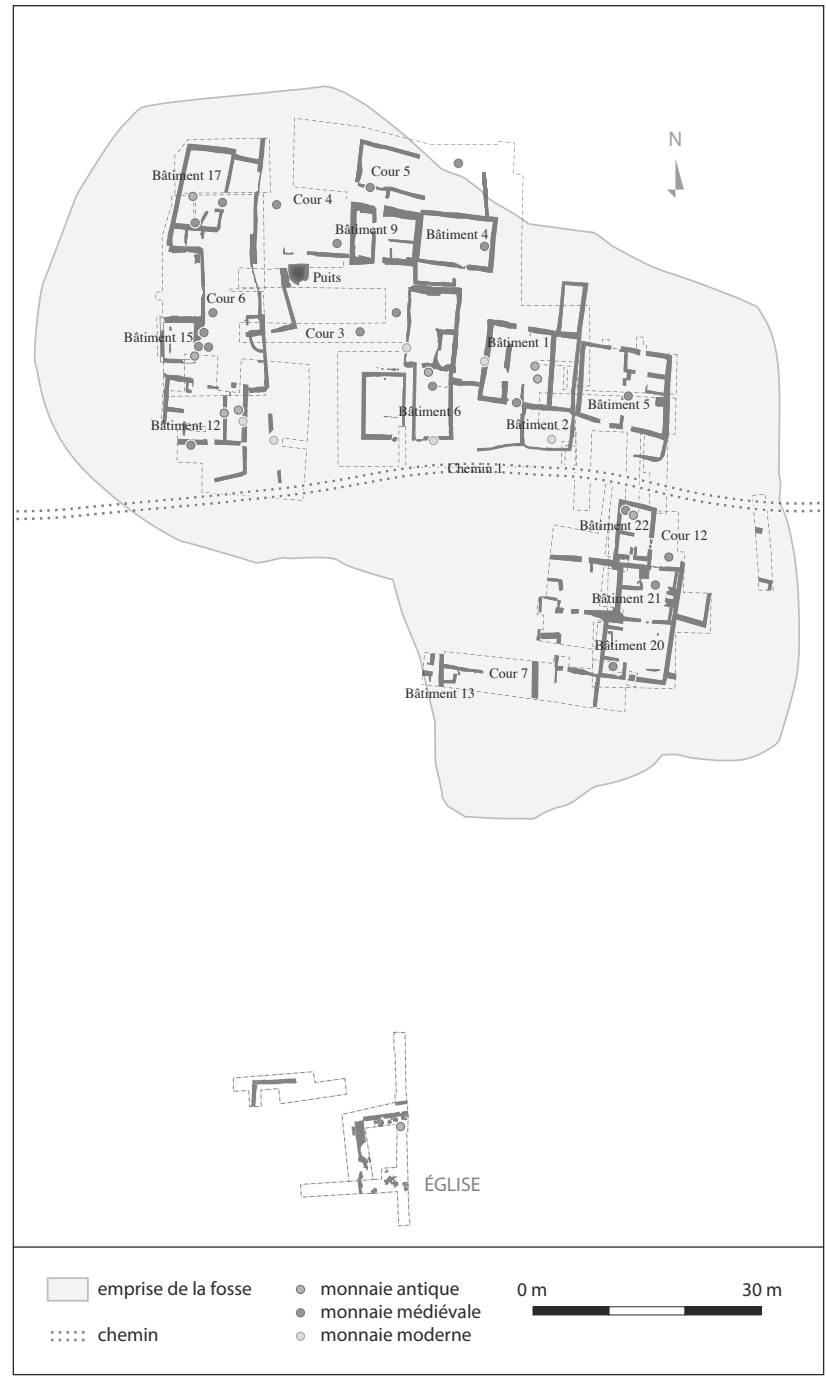

Figure 1 : (Voir planche couleur XIV) Répartition spatiale des découvertes monétaires à Courtisigny.

Figure 1: (See colour plate XIV) Distribution of the discoveries at Courtisigny.
À l'analyse, les découvertes isolées de Courtisigny méritent une attention supplémentaire. Dans l'ensemble, la part formée par les monnaies royales est majoritaire. Au fil des grandes périodes d'occupation du site, la tendance n'en est que plus remarquable (tableau 2). La monnaie du roi domine dès la phase $2 \mathrm{C}$, pour être exclusive au cours de la période suivante. Si le fait est à souligner, il ne doit pas surprendre. En effet, il n'est pas inutile de rapprocher cet ensemble de la composition de quelques trouvailles contemporaines, tels que les trésors de Louviers (Eure), SaintAubin-Épinay (Seine-Maritime), Pont-de-l'Arche (Eure) ou les découvertes isolées de Caen (Calvados), Saint-Vaastsur-Seulles (Calvados), Rouen (Seine-Maritime), Vattevillela-Rue (Seine-Maritime) ${ }^{4}$. La comparaison permet ainsi de constater que la prédominance des monnaies royales à Courtisigny se conforme à ce qui est généralement observé à l'échelle régionale au cours du XIve siècle. Toutefois, dans le détail, une variation est notable au cours de la période d'inflation des années 1350. Les imitations bretonnes (au nom de Charles de Blois) de monnaies royales comptent pour la moitié des découvertes. Il s'agit là d'une tendance qui se manifeste aussi sur d'autres sites bas-normands (tableau 5), ainsi que dans quelques trésors, comme ceux de Saint-Martin-de-Boscherville (Seine-Maritime; Salaün et Moesgaard, 1997) et de Louviers (Eure; Moesgaard, 2009, p. 267, T4). Pour J. Moesgaard, la situation s'expliquerait par un ralentissement, dans les ateliers royaux, de la frappe de la monnaie noire au profit de la monnaie blanche. Le duc de Bretagne aurait ainsi profité d'un espace laissé vacant par les ateliers royaux en introduisant dans la circulation des imitations destinées à lui procurer de l'argent pour la guerre qui l'opposait alors à Jean de Montfort'5.

Les vues globales auxquelles peut légitimement conduire la structure du lot monétaire laissent place à quelques constatations. Il y a lieu notamment de se demander quelles étaient les conditions concrètes dans lesquelles se trouvait placé à

\begin{tabular}{|l|l|l|}
\hline Monnaies en or & Monnaies blanches & Monnaies noires \\
\hline
\end{tabular}

\begin{tabular}{|c|c|c|c|}
\hline \multicolumn{4}{|l|}{ Basse-Normandie* } \\
\hline Courtisigny & \multirow[b]{3}{*}{1} & 4 & 16 \\
\hline Grentheville & & 18 & 45 \\
\hline Domfront & & 5 & 15 \\
\hline \multicolumn{4}{|l|}{ Haute-Normandie $^{\star *}$} \\
\hline Aizier & \multirow{5}{*}{1} & & 14 \\
\hline Fécamp & & 1 & 1 \\
\hline Grosley-sur-Risle & & 1 & 3 \\
\hline Louviers, rue Saint-Germain & & & 9 \\
\hline Rouen, place Foch & & & 34 \\
\hline
\end{tabular}

Tableau 4 : Découvertes de monnaies isolées en or, argent et en billon noir (début XIV ${ }^{\mathrm{e}}$ milieu XVI ${ }^{\mathrm{e}}$ siècle).

Table 4: Discoveries of isolated gold, silver and billon coins (beginning $14^{\text {th }}$-middle $16^{\text {th }}$ century).

*Base de données Nummus, service de numismatique, CRAHAM

**Monnaies de la période 1337-1577 (cf.

Moesgaard 2009, p. 234, tab. 6)

4. Pour la Seine-Maritime voir la synthèse de Moesgaard 2009, p. $265-$ 266 (T2, T4, T5), 270-273. Pour le Calvados : Base de données Nummus, service de numismatique Centre Michel-de-Boüard (université de Caen).

5. Idée résumée dans Moesgaard 2009, p. 249-250. Voir également Salaün 2000, p. 18. 


\begin{tabular}{|l|ccc|}
\cline { 2 - 4 } \multicolumn{1}{c|}{} & Monnaies royales & Bretagne & Autres \\
\hline Courtisigny & 2 & 3 & 1 \\
Grenthville, Trainecourt & 4 & 11 & 0 \\
Domfront & 4 & 5 & 0 \\
\hline
\end{tabular}

Tableau 5 : Répartition par pays d'origines des monnaies frappées au cours des années 1350 .

Table 5: Distribution by origin of the minted coins during the years 1350 .

cet égard l'ensemble. La première idée qui vient naturellement à l'esprit est de centrer l'attention sur le faciès global. L'importance quantitative du numéraire royal a été soulignée. Or, nous savons que l'une des conséquences de la conquête de la Normandie par Philippe II Auguste (1204) fut d'ouvrir progressivement la circulation régionale aux monnaies royales. Aussi parait-il indispensable de lier la structure globale du lot de Courtisigny aux effets de cet épisode. Dans un contexte de réglementation accrue, où la monnaie tend à devenir un privilège royal en France à l'image des modèles anglais et napolitain ${ }^{6}$, la part réduite prise par le monnayage baronnial n'est pas moins significative. Elle souligne en effet les efforts constants des princes à imposer dans la circulation leurs propres monnaies, ce qui entrainera la disparition presque totale des espèces seigneuriales (frappe et circulation) au début du XIV siècle (Duplessy, 1995, p. 12-13). Toute généralisation serait cependant excessive, comme le démontrent les découvertes de Courtisigny, et plus largement les découvertes normandes. Au cours des années 1350, il apparaît en effet que la Bretagne, pour tirer profit de l'insuffisance de l'approvisionnement monétaire royal, alimentait en monnaies la circulation régionale - bien que le duc de Bretagne fut déjà rappelé à l'ordre en 1339 (Bigot, 1857, p. 363-365).

\section{LA MONNAIE ET SON USAGE À CoURTISIGNY}

La rareté relative et le caractère accidentel des vestiges perdus sur place rendent nécessairement lacunaire l'interprétation du matériel monétaire de Courtisigny. Toutefois, celui-ci présente un intérêt intrinsèque réel sur lequel il faut encore insister : la topographie globale des vestiges dans l'espace, qui permet concrètement d'examiner la place réservée à la monnaie.

L'analyse de la carte de répartition souligne en premier lieu la large représentation du signe monétaire (figure 1). Bien qu'en faible nombre, la monnaie est attestée en plusieurs endroits du site. Il est ainsi frappant de voir que des découvertes ont été signalées éparses dans des couches d'oc-

6. Sur le sujet cf. Contamine (dir.), 1993, p. 266-267. cupation et en lien avec des niveaux de circulation ou de comblement. Ces caractéristiques générales sont peut-être tributaires des seules explorations archéologiques, mais plus vraisemblablement de la densité de l'occupation humaine. Tout de suite apparaissent deux traits dominants qui caractérisent fortement l'usage dont il était fait de la monnaie à Courtisigny au bas Moyen Âge.

Le premier, c'est son harmonieuse répartition, qui témoigne d'un certain dynamisme de la circulation monétaire. D'autre part, cette circulation était faite, comme nous l'avons vu, d'espèces de qualité médiocre, parfois détestable, et surtout de faible valeur. De caractère trop bon marché pour avoir une grande valeur unitaire, les monnaies noires de Courtisigny ne s'utilisaient que dans le cadre de petits achats. À l'évidence, leur utilisation n'était rendue possible que par le fait qu'elles étaient régulièrement manipulées à la campagne. Tout semble du moins l'indiquer. Pourtant, l'image d'une campagne rompue aux transactions monétaires quotidiennes a suscité quelques réserves. Pour P. Spufford (Spufford, 1988) notamment, les transactions impliquant des monnaies noires s'effectuaient surtout dans les villes, qui constitueraient le secteur le plus monétarisé de la société médiévale. À la campagne, la vie quotidienne des paysans aurait été alimentée par les denrées recueillies sur le sol dont on était propriétaire ou exploitant. Par conséquent, pour le petit nombre des échanges ordinaires, la manipulation d'espèces n’aurait été nullement nécessaire. Lorsque la monnaie avait lieu d'être utilisée, son usage devait se limiter à celui d'espèces en argent destinaient à des transactions exceptionnelles (vente de récoltes, paiement de l'impôt, etc.). Force est donc de constater que les découvertes de Courtisigny ne s'accordent pas avec cette image, ce que confirment d'ailleurs de récentes recherches (Bompaire, 2005; Moesgaard, 2005; Moesgaard, 2009, p. 259-260). Elles tendent au contraire à prouver que la monnaie était présente au quotidien parmi les paysans à la campagne pour le paiement comptant d'objets de consommation ou de certains services. Toutefois, le montant de chaque opération devait être extrêmement faible. Si faible que la monnaie d'argent et a fortiori la monnaie d'or représentait une unité trop forte. Son absence du village de Courtisigny s'expliquerait dès lors par le fait qu'elle ne trouvait pas sa place dans le cadre provincial. La monnaie saine et forte était une monnaie d'exportation, de la province vers la grande ville ou vers l'étranger pour paraphraser J. Meuvret (Meuvret, 1971, p. 134).

Si les découvertes de Courtisigny constituent donc une preuve forte d'une monétarisation en profondeur de la société paysanne, elles ne sont pas le seul exemple. Peu à peu, la vie économique des campagnes normandes se précise. Ainsi, les nombreuses monnaies noires mises au jour régulièrement par l'archéologie sur des villages du bas Moyen 
Âge (Grosley-sur-Risle, Trainecourt à Grentheville) ou sur quelques manoirs (Le Mesnil-sous-Jumièges, etc.), châteaux (Vatteville-la-Rue, Gavray, Saint-Vaast-sur-Seulles, etc.), abbayes (Saint-Martin-de-Boscherville, etc.) et autres léproseries (Bernay, etc.) de Basse et Haute-Normandie confirment de manière de plus en plus formelle l'utilisation courante de la menue monnaie parmi la population paysanne.

\section{Catalogue des monnaies Jacqueline Pilet- Lemière et Pierre-Marie Guihard}

\section{Ouvrages utilisés pour le classement}

Bastien, 1964 : Bastien P. - Le monnayage de Magnence, Wetteren, 1964.

LRBC : Carson R.A.G. et Kent J.P.C. - Late Roman

Bronze Coinage. Part I, The bronze coinage of the house of Constantine A.D. 324-346, London, 1972.

LRBC II : CARson R.A.G. et Kent J.P.C. - Late Roman Bronze Coinage. Part II, Bronze roman imperial coinage of the later Empire A.D. 346-498, London, 1972.

D : Duplessy J. - Les monnaies royales françaises de Hugues Capet à Louis XVI, vol. I, Paris, 1988 et vol. II, Paris, 1989.

Duplessy Féodales : DupLessy J. - Les monnaies françaises féodales, tome I, Paris, 2004.

L : LAfaurie J. - Les monnaies des rois de France, vol. I, Paris, 1951.

MEC : Grierson P. et Blackburn M. - Medieval European Coinage, I. The Early Middle Ages, Cambridge University Press, 1986.

PA I : Poey d’Avant F. - Monnaies féodales de France, vol. I, Paris, 1858.

PA III : Poey d’Avant F. - Monnaies féodales de France, vol. III, Paris, 1862.

Prou : Prou M. - Les monnaies carolingiennes, Paris, 1892.

Salaün 2000 : Salaün G. - Répertoire raisonné des monnaies de la guerre de succession de Bretagne, 1341-1365, SBNH, hors-série janvier, Vannes, 2000.

RIC I : Sutherland C.H.V. - The Roman Imperial Coinage, I. From 31 BC to AD 69, Londres, 1984.

RIC III : Mattingly H. et Sydenham E. A. - The Roman Imperial Coinage, III. Antoninus Pius to Commodus, Londres, 1930.

\section{Liste des abréviations}

dr. : droite

g. : gauche

*: Monnaie photographiée imit. : imitation

ind. : indéterminé

\section{Antiquité}

$1^{*}$. Néron, 54-68, Haut-Empire romain Avers : [IMP NERO] CAESAR AVG [P MAX TR P P P] Tête nue à g.; à la base du coup, un globe Revers: Anépigraphe // S C

Victoire à g., posant la main dr. sur un bouclier où se lit SPQR.

As (Ae); 7,57 g; $27 \mathrm{~mm}$

Lyon 54-68; RIC I, n 606, p. 185.

Inv. 71006 (nº d'étude : 6)

Nature activité : Occupation / seuil ; Contexte : Bâtiment 15 (32 V B 1); Datation contexte: XIII'-XIV ${ }^{\mathrm{e}}$ s.

2*. Antonin le Pieux, 138-161, Haut-Empire romain Avers : ANTONINVS AVG PIVS P P [...]

Tête laurée à dr.

Revers : [...] // S C

Aequitas (''Équité) debout à g., tenant une corne d'abondance dans la main g. et une balance dans la main $\mathrm{dr}$. Sesterce (Ae) ; 19,55 g; $30 \mathrm{~mm}$

Rome 148-161; RIC III, n ${ }^{\circ}$ 855, p. 133 pour le type. Inv. 72002 (n ${ }^{\circ}$ d'étude : 10)

Nature activité : Éboulis / occupation; Contexte : Bâtiment 22 (36 IV A2); Datation contexte : $\mathrm{XIV}^{\mathrm{e}}$ s. et après.

3. Indéterminée, Haut-Empire romain

Avers : [...]

Tête à $\mathrm{g}$.

Revers : [...]

Personnage assis à g. (?)

As (Ae); $9,57 \mathrm{~g} ; 24 \mathrm{~mm}$

Atelier ind. 100-199.

Inv. 50440007 ( $\mathrm{n}^{\circ}$ d'étude : 50)

US couche : 5044; Nature activité : Occupation; Contexte : Bâtiment 12 / foyer 8; Datation contexte : $\mathrm{xIV}^{\mathrm{e}} \mathrm{s}$.

4. Constantin I ${ }^{\mathrm{er}}$, 307-337, Bas-Empire romain Avers : [CONSTAN]-TINVS A[VG]

Tête laurée à dr.

Revers : [PR]OVIN[DEN-TIAE AVGG] // PTR[...] Porte de camp avec deux tours, au-dessus une étoile.

Nummus (Ae); 3,00 g; 17 mm

Trèves 324-330; $L R B C \mathrm{I}, \mathrm{n}^{\circ} 12$, p. 4.

Inv. 11330001 ( $\mathrm{n}^{\circ}$ d'étude : 23) 
US couche : 1133; Nature activité : Occupation / sol; Contexte : Bâtiment $1 /$ pièce 2 ; Datation contexte : $\mathrm{XIV}^{\mathrm{e}} \mathrm{s}$.

$5^{*}$. Époque constantinienne, Bas-Empire romain

Avers : [...]

Buste diadémé à dr.

Revers : [GLOR-IA] EXER-CITVS

Deux soldats se faisant face, tenant une haste renversée et posant la main sur un bouclier; entre eux, une enseigne.

Nummus (Ae); 1,34 g; 15 mm

Atelier ind. 335-341.

Inv. $50550001\left(\mathrm{n}^{\circ}\right.$ d'étude : 48)

US couche : 5055; Nature activité : Éboulis; Contexte :

Bâtiment 12 / pièce 2 ; Datation contexte : $\operatorname{XIV}^{\mathrm{e}} \mathrm{s}$.

6. Époque constantinienne, Bas-Empire romain

Avers : Illisible. Type Constantinopolis

Buste casqué à g. avec aigrette.

Revers : Anépigraphe // [...]

Victoire debout à g. sur une proue, tenant un sceptre long et un bouclier.

Nummus (Ae); 1,76 g; $18 \mathrm{~mm}$

Atelier ind. 330-341.

Inv. 11810027 ( ${ }^{\circ}$ d'étude : 32)

US couche : 1181; Nature activité : Occupation;

Contexte : Bâtiment 1 / foyer; Datation contexte : XIv s.

7. Indéterminée, Bas-Empire romain

Avers : [...]

Buste diadémé à dr.

Revers : [...] Type Victoriae dd augg q nn

Deux Victoires se faisant face et tenant chacune une couronne de laurier.

Nummus (Ae); 0,77 g; 13 mm

Atelier ind. 341-346

Inv. 71001 (nº d'étude : 2)

Nature activité : Occupation sol; Contexte : Cour 6 (32

VI D 1); Datation contexte : XIV es.

8*. Magnence, 350-353, pour Décence César, 351-353, Bas-

Empire romain

Avers : [D N DEC]ENTIVS CAESAR

Buste cuirassé à dr.; tête nue derrière laquelle figure la lettre A.

Revers : [VICTORIA]E DD NN AVG ET CAE / F // [S]AR

Deux victoires se faisant face, portant un bouclier sur lequel est portée la légende VOT/V/MVL/X.

Maiorina (Ae); 4,44 g; $20 \mathrm{~mm}$
Arles 351-352; $L R B C$ II, $\mathrm{n}^{\circ}$ 436, p. 55; Bastien 1964, $\mathrm{n}^{\circ} 264$, p. 187.

Inv. 20150032 ( $\mathrm{n}^{\circ}$ d'étude : 22)

US couche : 2015; Nature activité : Éboulis; Contexte :

Bâtiment 6; Datation contexte : XIV s.

9. Époque théodosienne, Bas-Empire romain

Avers : [...]VS PF AVG

Buste diadémé à dr.

Revers : SALVS REI[PVBLICAE]

Victoire à g., un trophée sur l'épaule, tirant un captif; un chrisme dans le champ à g.

Nummus (Ae); 1,07 g; $11 \mathrm{~mm}$

Imitation 388-395.

Inv. 40000000 ( $\mathrm{n}^{\circ}$ d'étude : 39)

US couche : 2; Nature activité : Aucune; Contexte : Humus / bâtiment 12; Datation contexte : XviII ${ }^{\mathrm{e}}$ s. et après.

\section{Époque carolingienne}

10*. Louis le Pieux, 814-840, Empire carolingien

Avers : + H LVD[OVVICVS IM]P

Croix cantonnée de quatre globules.

Revers : [XPSTI]ANA REL[IGIO]

Temple tétrastyle sur deux degrés.

Denier (Ag); 0,66 g; $20 \mathrm{~mm}$

Atelier ind. 814-840; Prou 987 et suiv.; MEC class 3, p. 520.

Inv. 72003 (nº d'étude : 11)

Nature activité : Occupation / sol; Contexte : Bâtiment

21 ; Datation contexte : $\mathrm{XIV}^{\mathrm{e}} \mathrm{s}$.

\section{Moyen Âge / Royaume de France}

11*. Louis VIII, 1223-1226, et Louis IX, 1226-1270, Royaume de France

Avers : + LVD[OVICVS RE]X

Croix.

Revers : [+ TVRON]VS CIVI

Châtel tournois.

Denier tournois $(\mathrm{Ag}) ; 0,42 \mathrm{~g} ; 19 \mathrm{~mm}$

Tours 1223-1245; L 195; D 187.

Inv. 21760005 ( $\mathrm{n}^{\circ}$ d'étude : 43)

US couche : 2176; Nature activité : Occupation / sol;

Contexte : Cour 4; Datation contexte: XIII ${ }^{\mathrm{e}} \mathrm{XIV}^{\mathrm{e}} \mathrm{s}$.

12*. Philippe IV, 1285-1314, Royaume de France

Avers : + PH[ILIPP]VS REX

Croix feuillue.

Revers : + MONETA DVPLEX 
REGA/LIS sous une fleur de lis.

Double parisis (billon noir); 1,21 g; $20 \mathrm{~mm}$

Atelier ind. 1295-1303; L 232; D 227.

Inv. 71007 ( $\mathrm{n}^{\circ}$ d'étude : 7)

Nature activité : Occupation / sol; Contexte : Bâtiment 17 (33 VI-A4); Datation contexte : XIII ${ }^{\mathrm{e}}-\mathrm{XIV}^{\mathrm{e}}$ s.

13*. Philippe IV, 1285-1314, Royaume de France

Avers : Anépigraphe

Croix cantonnée de P, H', R, EX

Revers: Anépigraphe

Fronton de châtel tournois, annelé à sa base, sommé d'une croisette et accosté de deux lis.

Tournois simple (billon noir); 0,58 g; $15 \mathrm{~mm}$

Atelier ind. 1295-1303; L 235; D 230.

Inv. 72001 ( $\mathrm{n}^{\circ}$ d'étude : 9)

Nature activité : Éboulis / abandon; Contexte : Bâtiment

22 (36 IV-A 1); Datation contexte : XIV s. et après.

$14^{*}$. Philippe IV, 1285-1314, Royaume de France

Avers : + PHILIPPVS REX

Croix latine coupant la légende en bas.

Revers : BVRGENSIS

NOV/VS sous un lis accosté de deux annelets.

Bourgeois simple (billon noir); 0,69 g; $19 \mathrm{~mm}$

Atelier ind. 26 janvier 1311; L 237; D 232.

Inv. 72005 ( $\mathrm{n}^{\circ}$ d'étude : 13)

Nature activité : Occupation / sol; Contexte : Bâtiment

20 / pièce 2 ; Datation contexte : $\operatorname{XIV}^{\mathrm{e}} \mathrm{s}$.

15*. Philippe IV, 1285-1314, Royaume de France

Avers : + PHILIP/PVS REX

Croix latine tréflée, coupant la légende en bas.

Revers : BVRGENSIS

FOR/TIS en deux lignes, sous une couronne

Bourgeois fort (billon noir); $1,18 \mathrm{~g} ; 20 \mathrm{~mm}$

Atelier ind. 26 janvier 1311; L 236; D 231.

Inv. 21600016 ( ${ }^{\circ}$ d'étude : 38)

US couche : 2160; Nature activité : Occupation / sol; Contexte : Bâtiment 4 / proche foyer 5; Datation contexte : $\mathrm{XIV}^{\mathrm{e}} \mathrm{s}$.

16. Philippe IV, 1285-1314, Royaume de France

Avers : + PHILIP/PVS REX

Croix latine coupant la légende en bas.

Revers : BVRGENSIS

NOV/VS sous un lis accosté de deux annelets.

Obole bourgeoise (Ag); 0,49 g; $15 \mathrm{~mm}$

Atelier ind. 26 janvier 1311; L 238; D 233.

Inv. 21860055 (nº́d'étude : 42)
US couche : 2186; Nature activité : Occupation / sol;

Contexte : Cour 4; Datation contexte : XIII'-XIV ${ }^{\mathrm{e}}$ s.

17. Charles IV, 1322-1328 ou Philippe VI, 1328-1350, Royaume de France

Avers : [...] R[EX]

Couronne.

Revers : + MON[ETA DVPLEX]

Croix fleurdelisée.

Double Parisis (Billon noir); 0,70 g; $21 \mathrm{~mm}$

Atelier ind. 1322-1328; L 248 ou L 269 / D 244b et c ou D 266.

Inv. 71004 (nº d'étude : 3)

Nature activité : Aucune / humus; Contexte : Bâtiment 5

(33 V-A 1); Datation contexte : XIv ${ }^{e}$ s. et après.

18*. Philippe VI, 1328-1350, Royaume de France

Avers : + PHILIPPVS REX

Croix.

Revers : + F[RANCO]RVM

Châtel tournois sans meubles.

Pite tournois (billon blanc); 0,45 g; $16 \mathrm{~mm}$

Atelier ind. 6 septembre 1329; L 288; D 280.

Inv. 73001 ( $n^{\circ}$ d'étude : 14)

Nature activité : Occupation; Contexte : Bâtiment 5;

Datation contexte : $\mathrm{XIV}^{\mathrm{e}} \mathrm{s}$.

19*. Philippe VI, 1328-1350, Royaume de France

Avers : (couronne) [PHILIPPVS] (trèfle) [REX]

FRA/NCO en deux lignes.

Revers : (couronne) MONETA (trèfle) DVPLEX

Croix fleurdelisée.

Double Parisis (Billon noir); 1,03 g; $21 \mathrm{~mm}$

27 avril 1346; L 271; D 269

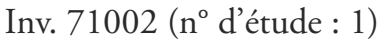

Nature activité : Occupation; Contexte : Cour 6 (31 VI

B 3); Datation contexte : $\mathrm{XIII}^{\mathrm{e}}-\mathrm{XIV}^{\mathrm{e}} \mathrm{s}$.

20*. Philippe VI, 1328-1350, Royaume de France

Avers : (lis) PHILIPPVS REX

FRAN/CORV en deux lignes.

Revers : + MONETA DVPLEX

Croix latine dont les trois bras supérieurs sont fleurdelisés.

Double parisis (billon noir); 0,78 g; $23 \mathrm{~mm}$

Atelier ind. 21 août 1350; L 272a; D 270A.

Inv. 30720001 ( $\mathrm{n}^{\circ}$ d'étude : 45)

US couche : 3072; Nature activité : Occupation / mur

61; Contexte : Cour 5; Datation contexte : $\mathrm{XIV}^{\mathrm{e}}-\mathrm{XV}^{\mathrm{e}}$ s.

21*. Jean II, 1350-1364, Royaume de France

Avers : + IOH[ANNES FRANCORV R]EX 
Fleur de lis.

Revers : + [MONETA DVPLEX]

Croix latine fleurdelisée et recroisetée, coupant la légende en bas.

Double tournois (billon noir); 1,26 g; $22 \mathrm{~mm}$

Atelier ind. 7 février 1358; L 334; D 324.

Inv. 72004 ( $n^{\circ}$ d'étude : 12)

Nature activité : Occupation / sol; Contexte : Cour 12 (36 IV); Datation contexte : $\mathrm{XIV}^{\mathrm{e}} \mathrm{s}$.

22*. Louis XI, 1461-1483, Royaume de France

Avers : + LVDOVICVS xREX

Couronne.

Revers : OBO/LVS/xCI/VIS

Croix coupant la légende.

Obole tournois (billon noir); 0,42 g; $14 \mathrm{~mm}$

Atelier ind. 20 novembre 1467; L 549a; D 565.

Inv. 21630050 ( ${ }^{\circ}$ d'étude : 40)

US couche : 2163; Nature activité : Occupation / aban-

don; Contexte : Cour 4; Datation contexte $: \mathrm{Xv}^{\mathrm{e}} \mathrm{s}$.

23*. Louis XI, 1461-1483, Royaume de France

Avers : Légende hors flan. Type Ludovicus franc rex

Deux lis dans un trilobe, ponctué d'annelets.

Revers: (lis) SIT NO[...]VM

Croix dans un quadrilobe, ponctué d'annelets.

Denier tournois (billon noir); 0,93 g; $16 \mathrm{~mm}$

Atelier ind. 15 septembre 1476; L 547a; D 563A.

Inv. $20380012\left(\mathrm{n}^{\circ}\right.$ d'étude : 24)

US couche : 2038; Nature activité : Éboulis; Contexte :

Bâtiment 6; Datation contexte : $\mathrm{XIV}^{\mathrm{e}} \mathrm{s}$.

24*. François I ${ }^{\text {er }}$, 1515-1547, Royaume de France

Avers : + FRAN [...]

Trois lis posés 2 et 1.

Revers : [...]BEN[...]

Croix plaine.

Double tournois (Billon noir); 0,58 g; $17 \mathrm{~mm}$

Atelier ind. 19 mars 1541; D 935; L 791.

Inv. 10740004 ( ${ }^{\circ}$ d'étude : 18)

US couche : 1074; Nature activité : Éboulis / Occupation;

Contexte : Bâtiment 2; Datation contexte : Post XIv ${ }^{\mathrm{e}} \mathrm{s}$.

25*. Henri II, 1547-1559, Royaume de France

Avers : [+ HENRI 2 D G FRAN] REX

Trois lis posés 2 et 1 , sans trilobe.

Revers : [+ SIT NOMEN DNI] BENE 1552

Croix plaine dans un quadrilobe. À l'exergue, lettre X, sous la croix.

Double tournois (billon noir); 0,52 g; $14 \mathrm{~mm}$

Villefranche-de-Rouergue 1552; D 1005A.
Inv. 20060018 ( $\mathrm{n}^{\circ}$ d'étude : 21 )

US couche : 2006; Nature activité : Éboulis / occupation;

Contexte : Bâtiment 6 / cheminée 1; Datation contexte : $\mathrm{XIV}^{\mathrm{e}} \mathrm{s}$.

26. Louis XIII, 1610-1643, Royaume de France Avers : [LOYS XIII R DE] FRAN ET NAV

Grand buste à dr., drapé et cuirassé.

Revers : + DOVBLE [TOVRNOIS]

Trois posés 2 et 1 .

Double tournois $(\mathrm{Cu}) ; 2,40 \mathrm{~g} ; 20 \mathrm{~mm}$

Atelier ind. émission postérieure à 1635; D 1371.

Inv. 50090006 ( $\mathrm{n}^{\circ}$ d'étude : 44)

US couche : 5009 .

27. Louis XIII, 1610-1643, Royaume de France

Avers : [LOYS XIII R DE] FRAN ET NAV

Buste à dr

Revers : + DOVBLE TOVRNOIS

Trois lis posés 2 et 1 .

Double tournois $(\mathrm{Cu}) ; 2,40 \mathrm{~g} ; 20 \mathrm{~mm}$

Atelier ind. 1600-1699.

Inv. 50090006 ( $\mathrm{n}^{\circ}$ d'étude : 47)

Nature activité : Tranchée de récupération / mur 64;

Contexte : Cour 6; Datation contexte : XviI ${ }^{\mathrm{e}}$ s. et après.

28. Louis XIV, 1643-1715, Royaume de France

Avers : L XIIII ROY DE [FR ET DE NA]

Buste juvénile du roi à dr., couronné, drapé et cuirassé.

Revers : [LIARD DE FRANCE en trois lignes]

Différent d'atelier illisible qui est entouré de trois lis posés

2 et 1.

Liard de France $(\mathrm{Cu}) ; 2,83 \mathrm{~g} ; 23 \mathrm{~mm}$

Atelier ind. 1655-1658; D 1588.

Inv. 10330001 ( $\mathrm{n}^{\circ}$ d'étude : 15$)$

Nature activité : Abandon; Contexte : Bâtiment 1;

Datation contexte : Post-médiéval

29*. Louis XIV, 1643-1715, Royaume de France

Avers : L XIIII ROY DE FR ET DE NA 1658

Buste à dr. du roi, couronné, drapé et cuirassé.

Revers : LIARD/DE/FRANCE

Différent d'atelier (lettre E) entouré de trois lis posés 2 et 1 .

Liard de France $(\mathrm{Cu}) ; 3,30 \mathrm{~g} ; 22 \mathrm{~mm}$

Chatellerault 1658; D 1588.

Inv. 21380023 ( $\mathrm{n}^{\circ}$ d'étude : 36)

US couche : 2138; Nature activité : Aucune; Contexte :

Humus; Datation contexte : Postérieur $\mathrm{Xv}^{\mathrm{e}} \mathrm{s}$.

30*. Louis XIV, 1643-1715, Royaume de France 
Avers : LVD XIIII D G FR ET N R

Croix formée de huit $\mathrm{L}$ adossées, chaque bras étant couronné, avec différent d'atelier (ici illisible) dans un cercle en cœur, cantonnée de quatre lis divergeant du centre.

Revers : SIT NOMEN DOMINI BENEDICTVM 1694

Écu de France couronné, contremarqué d'un lis.

Quinzain (Billon); 1,47 g; $24 \mathrm{~mm}$

Atelier ind. 1694; D 1581A.

Inv. 50010028 ( ${ }^{\circ}$ d'étude : 46)

US couche : 5001; Nature activité : Aucune; Contexte :

Humus / bâtiment 12; Datation contexte : XIV ${ }^{\mathrm{e}}$ s. et après.

31*. Gaston d'Orléans, 1627-1650 pour Anne-Marie-

Louise, 1650-1693, Royaume de France

Avers : GASTON V [F P D]

Buste à dr. lauré.

Revers : [+ DE]NIER TOVRNOIS 1655

Deux lis.

Denier tournois $(\mathrm{Cu}) ; 1,47 \mathrm{~g} ; 16 \mathrm{~mm}$

Trévoux 1655; PA III, n 5214.

Inv. 20010002 (nº d'étude : 16)

Nature activité : Aucune; Contexte : Humus; Datation

contexte : postérieur $\mathrm{XIV}^{\mathrm{e}} \mathrm{s}$.

\section{Moyen Âge / Monnayage baronnial}

32*. Erbert I ${ }^{\mathrm{er}}$, 1015-1036, Comté du Mans

Type immobilisé

Avers : + COMES CENOMANIS (ligature entre M et E de COMES)

Monogramme d'Erbert.

Revers : + SIGNVM DEI VIVI

Croix cantonnée d'un besant aux 1 et 2, de l'alpha et de l'oméga aux 4 et 3 .

Denier (Ag), 0,92 g; $20 \mathrm{~mm}$

Le Mans 1080-1130; PA I, n 1552; Duplessy Féodales, $\mathrm{n}^{\circ} 399$.

Inv. $11030001\left(\mathrm{n}^{\circ}\right.$ d'étude : 20)

US couche : 1103; Nature activité : Éboulis / occupation; Contexte : Bâtiment 1 / seuil 2 (bouchage de la porte); Datation contexte : $x v^{e} s$.

33*. Anonyme, Comté de Vendôme

Avers : Anépigraphe

Tête bléso-chartraine à dr., accostée d'un annelet à g. et d'un croissant entre deux annelets à dr., avec une croisette en cœur et $S$ et trois traits au-dessous.

Revers : + VDON CAOSTO

Croix.

Denier (Ag); 0,74 g; $18 \mathrm{~mm}$

Vendôme 1180-1205; PA I, n 1779; D 2004, n 545.
Inv. 30190033 ( $n^{\circ}$ d'étude : 35)

US couche : 3019; Nature activité : Aucune; Contexte :

Humus; Datation contexte : $\mathrm{XIV}^{\mathrm{e}}-\mathrm{XX}^{\mathrm{e}} \mathrm{s}$.

34*. Anonyme, Vicomté de Châteaudun

Avers : Anépigraphe

Tête bléso-chartraine à g., entre un besant à dr. et un croissant à g. et une étoile entre deux points au-dessous.

Revers : + CASTRI DVRI

Croix.

Denier (Ag); 0,78 g; $19 \mathrm{~mm}$

Châteaudun 1200-1210; PA I, n 1845; Duplessy

Féodales, $n^{\circ}$ 488A.

Inv. 10890002 ( $\mathrm{n}^{\circ}$ d'étude : 19)

US couche : 1050; Nature activité : Éboulis / occupation;

Contexte : Bâtiment 2; Datation contexte : $c a$. milieu $\mathrm{XIV}^{\mathrm{e}} \mathrm{s}$.

35*. Charles de Blois, 1341-1364, Duché de Bretagne

Avers : + KA[ROLVS DV]X

Croix.

Revers : [+ B]RI[TANNORVM]

Châtel tournois avec meuble.

Denier tournois (billon noir) / imitation monnaie française; $0,58 \mathrm{~g} ; 17 \mathrm{~mm}$

Atelier ind. ca 1354-1355; Salaün 2000, p. 44.

Inv. 71003 (nº d'étude : 4)

Nature activité : Occupation / sol; Contexte : Bâtiment 15 (33 V B 1); Datation contexte : $\mathrm{XIV}^{\mathrm{e}}$ s.

36*. Charles de Blois, 1341-1364, Duché de Bretagne

Avers : + KAROL $\mathrm{VS}^{\circ} \mathrm{R}^{\circ} \mathrm{DVX}$

Croix.

Revers : + BR[ITANNORVM]

Illisible

Denier tournois (billon noir) / imitation monnaie française; $0,48 \mathrm{~g} ; 20 \mathrm{~mm}$

Rennes 1354; Salaün 2000, p. 45

Inv. 71005 ( $\mathrm{n}^{\circ}$ d'étude : 5)

Nature activité : Occupation; Contexte : Bâtiment 12 (33

V-B 1); Datation contexte : XIV s.

37*. Charles de Blois, 1341-1364, Duché de Bretagne

Avers : [+ KAROLVS B]RITANORV

Dans le champ, DUX entre deux rangs de pseudo-lis, ceux du bas étant à l'envers.

Revers : [+ MONETA DVPLEX]

Croix égale, ayant à chaque extrémité trois bourrelets inégaux.

Double tournois (Billon noir) / imitation monnaie française; $0,69 \mathrm{~g} ; 19 \mathrm{~mm}$ 

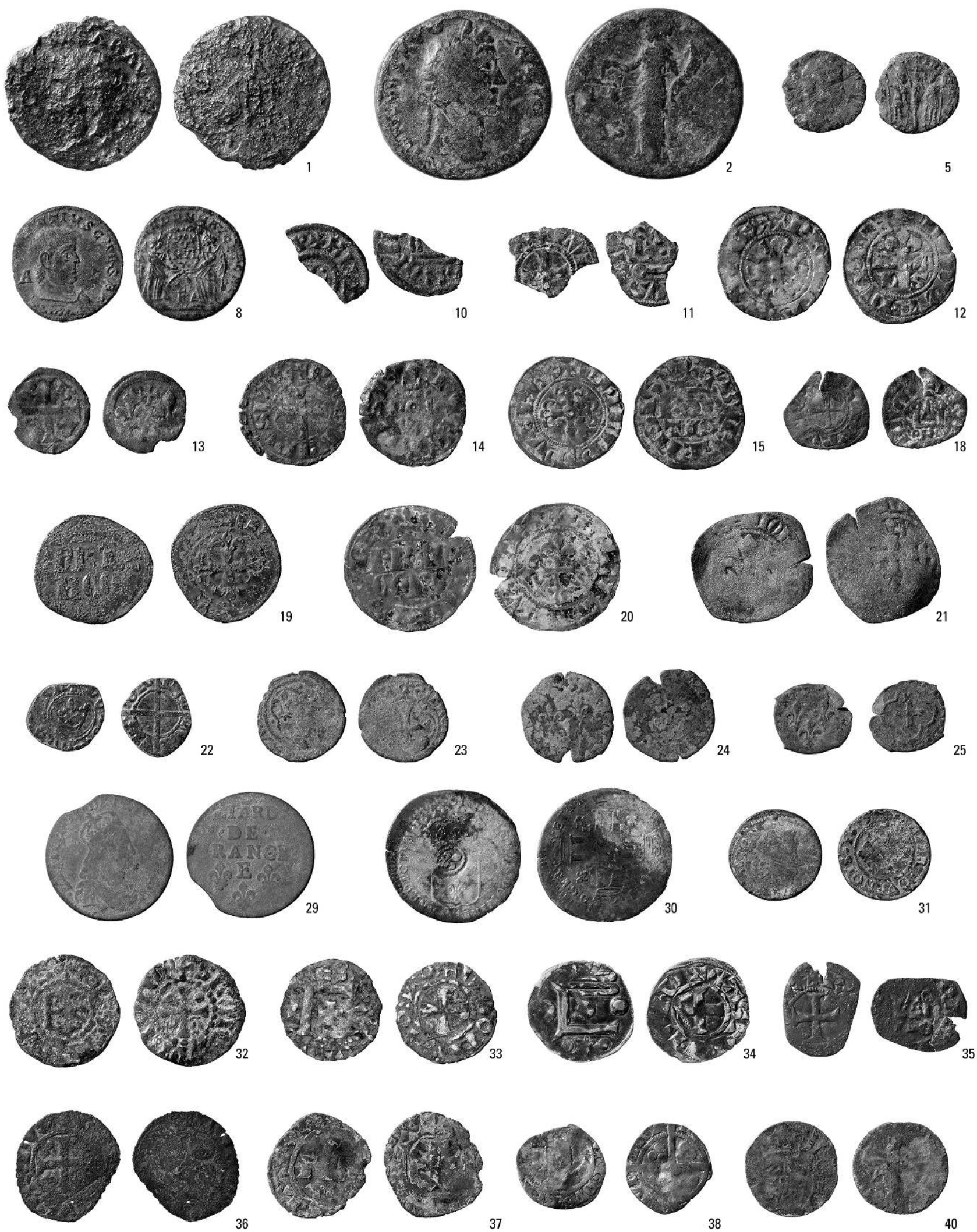
Atelier ind. 1356; PA I, n 520; Salaün 2000, n 209; Duplessy Féodales, $\mathrm{n}^{\circ} 142 \mathrm{~A}$.

Inv. $50680022\left(\mathrm{n}^{\circ}\right.$ d'étude : 49)

US couche : 5068; Nature activité : Occupation / dépotoir; Contexte : Bâtiment 12 / pièce 4; Datation contexte : $\mathrm{XIV}^{\mathrm{e}} \mathrm{s}$.

\section{Moyen Âge / Royaume d'Angleterre}

38*. Edouard II, 1327-1377, Royaume d'Angleterre

Avers : [EDW]ARDVS REX [ANGLI]

Tête de face, presque illisible.

Revers : $\mathrm{LO}[\mathrm{ND} / \mathrm{ON}] \mathrm{CIVI} / \mathrm{TAS}$

Croix longue coupant la légende cantonnée de trois besants dans chaque canton.

Denier (Ag); 0,70 g; $16 \mathrm{~mm}$

Londres 1351-1361; North II, p. 36-41.

Inv. 20880056 ( ${ }^{\circ}$ d'étude : 31)

US couche : 2088; Nature activité : Occupation / sol;

Contexte : Cour 3; Datation contexte : $\mathrm{XIII}^{\mathrm{e}}-\mathrm{XX}^{\mathrm{e}} \mathrm{s}$.

\section{Moyen Âge}

39. Indéterminée

Avers : [...]

Croix.

Revers : [...]

Illisible.

Denier (billon noir); 0,45 g; $17 \mathrm{~mm}$

Atelier ind. ca. 1350.

Inv. 71008 (nº d'étude : 8)

Nature activité : Occupation / seuil ; Contexte : Bâtiment

17 / seuil (33 VI-A4); Datation contexte : XIII ${ }^{\mathrm{e}}$-XIVe s.

40*. Indéterminée

Avers : [...]

Écusson (?)

Revers : [...]

Croix.

Denier (billon blanc); 0,66 g; $17 \mathrm{~mm}$

Atelier ind. 1300-1399.

Inv. 210030041 ( $\mathrm{n}^{\circ}$ d'étude : 33)

US couche : 2103; Nature activité : Occupation / sol;

Contexte: Cour 3; Datation contexte : $\mathrm{XIII}^{\mathrm{e}}-\mathrm{XX}^{\mathrm{e}} \mathrm{s}$.

\section{Bibliographie}

Bigot A., I 857 - Essai sur les monnaies du royaume et duché de Bretagne, Paris.

Bompaire M., I999 - " Pour une histoire des monnaies noires ", in Kerhervé J. et Rigaudière A. (éd.), Finances, pouvoirs et mémoire, mélanges offerts à Jean Favier, Paris, p. 349-371.

Bompaire M., 2005 - "Monnaies dans les villages, quelques exemples de France méridionale ", in Lefort J., Morrisson C. et Sodini J.-P. (dir.), Les villages dans l'Empire byzantin IVe$X V^{e}$ siècle, Paris, p. 123-134.

CAG 14 - Delacampagne F., i990, Carte archéologique de la Gaule, «Le Calvados ", Paris.

Caumont (DE) A., I846 - Statistique monumentale du Calvados, Caen.

Contamine P. (dir.), I993 - L'économie médiévale, Paris.

Démians d’Archimbaud G., I980 - Les fouilles de Rougiers (Var). Contribution à l'archéologie de l'habitat rural médiéval en pays méditerranéen, Paris, Valbonne.

Depeyrot G., I976 - « Problèmes de réutilisation des monnaies romaines à Cahors (Lot) ", Bulletin de la Société française de Numismatique, t. 31, p. 11-12.

Doranlo R., i9r3-I9I4 - "Camps, enceintes, mottes et fortifications antiques du Calvados ", Bulletin de la Société des Antiquaires de Normandie, 29, p. 411-412.

Duplessy J., I 995 - Les trésors monétaires médiévaux et modernes découverts en France, t. II, Paris.

Hanusse C., 2006 - "L'organisation des espaces de circulation dans l'habitat rural de la plaine de Caen. L'exemple du site des Fosses Saint-Ursin (XIII ${ }^{\mathrm{e}}$-XIv ${ }^{\mathrm{e}}$ siècles) ", in AleXAndre-Bidon D., Piponnier F. et Poisson J.-M. (éd.), Cadre de vie et manières d'habiter, Caen, p. 81-89.

Meuvret J., I97 I - «Circulation monétaire et utilisation économique de la monnaie dans la France du Xvi et du XVII ${ }^{\mathrm{e}}$ siècle ", in Études d'histoire économique. Recueil d'articles, Paris, p. 127137.

MoesgaArd J.-C., 2005 - "Monnaies à la campagne au Moyen Âge, remarques de méthode ", in Lefort J., Morrisson C. et Sodini J.-P. (dir.), Les villages dans l'Empire byzantin IVe$X V^{*}$ siècle, Paris, p. 135-148.

MoesgaArd J.-C., 2009 - «La circulation des monnaies noires en Haute-Normandie, 1337-1577 ", Revue Numismatique, p. 221-305.

SALAÜN G., 2000 - Répertoire raisonné des monnaies de la succession de Bretagne 1341-1365, Nantes.

SAlaün G. et MoesgaARd J.-C., I 997 - « Un trésor du XIV ${ }^{\mathrm{e}}$ siècle près de Saint-Martin-de-Boscherville (Seine-Maritime) ", Bulletin de la Société Française de Numismatique, avril, p. 59-63.

Spufford P., I 988 - Money and its use un medieval Europe, Cambridge. 
Zusammenfassung: Münzen im ländlichen Raum: Die Funde aus dem mittelalterlichen Dorfvon Courtisigny „Les Fosses Saint-Ursin“ bei Courseulles-sur-Mer (Calvados, Normandie, Frankreich) - In den Jahren 1970 und 2000 haben die archäologischen Ausgrabungen im Bereich des mittelalterlichen Dorfes von Courtisigny „Les fosses Saint-Ursin“ bei Courseulles-sur-Mer (Calvados, Normandie, Frankreich) gestattet, ein Fundensemble antiker, mittelalterlicher und moderner Münzen zu bergen. Diese Funde wurden einer näheren archäologischen Analyse unterzogen, die dazu beiträgt, ihre Verbreitung und Zirkulation innerhalb des zentralen Dorfbereiches zu erfassen. Durch die Kombination numismatischer und archäologischer Quellen ist es möglich, die Frage der Münzeinführung im ländlichen Raum zwischen dem 12. und dem 14. Jahrhundert näher zu untersuchen.

Resumen: Monedas en medio rural: los descubrimientos de la aldea de Courtisigny ("Las fosas Saint-Ursin”, Courseulles-sur-Mer, Calvados) - En los años 1970 y 2000, las excavaciones del municipio medieval de Courtisigny ("Las fosas Saint-Ursin", Courseulles-sur-Mer, Calvados) destacaron un conjunto de monedas antiguas, medievales y modernas. Estos hallazgos han sido objetos de un estudio arqueológico que contribuyo a una mejor comprensión de su circulación en el centro del pueblo. Fue a partir del cruce de datos numismáticos y arqueológicos que fue posible abordar el tema de la monetización rural entre los siglos XII y XIV.

Schlüsselwörter: Münzen, Mittelalter, Archäologie, Münzumlauf, Münzeinführung im ländlichen Raum.

Palabras clave: Monedas, Edad Media, arqueología, circulación monetaria, monetización rural. 